Check for updates

Cite this: RSC Adv., 2017, 7, 22587

\title{
Atomic scale investigation of enhanced ferroelectricity in $(\mathrm{Ba}, \mathrm{Ca}) \mathrm{TiO}_{3} \uparrow$
}

\begin{abstract}
Xiao Na Zhu, ${ }^{a}$ Xing Chen, ${ }^{b}$ He Tian*b and Xiang Ming Chen (D) *a
The precise atomic positions and occupancies in $(\mathrm{Ba}, \mathrm{Ca}) \mathrm{TiO}_{3}$ ceramics have been characterized by high angle annular dark field (HADDF)-scanning transmission electron microscopy (STEM), electron energy loss spectroscopy (EELS), and annular bright field (ABF)-STEM. EELS precisely determines the distribution of $\mathrm{Ba}, \mathrm{Ca}$, and $\mathrm{Ti}$ ions in the perovskite structure, confirming the occupancy of $\sim 7 \% \mathrm{Ca}$ content at $\mathrm{B}$-site in $\left(\mathrm{Ba}_{0.75} \mathrm{Ca}_{0.25}\right) \mathrm{TiO}_{3}$, which is the first direct evidence of $\mathrm{Ca}$ occupancy at the $\mathrm{B}$-site in $(\mathrm{Ba}, \mathrm{Ca}) \mathrm{TiO}_{3}$ ceramics. ABF-STEM mapped the displacement of Ti ions and oxygen ions with respect to the center of $\mathrm{Ba}$ ions. By averaging the displacements of $\mathrm{Ti}$ and $\mathrm{O}$ ions in multiple single domains, it confirms the average Ti displacement of $0.263 \AA$, and large apical and equatorial oxygen displacement of $0.264 \AA$ and $0.347 \AA$, respectively. Such great displacements in $(\mathrm{Ba}, \mathrm{Ca}) \mathrm{TiO}_{3}$ compared to $\mathrm{BaTiO}_{3}$ are direct evidence for the structure origin of the ferroelectric enhancement in $\mathrm{Ca}$ substituted $\mathrm{BaTiO}_{3}$
\end{abstract}

Received 16th January 2017 Accepted 12th April 2017

DOI: $10.1039 / \mathrm{c} 7 \mathrm{ra00662d}$

rsc.li/rsc-advances domain structure, ${ }^{11-13}$ interface effect ${ }^{14}$ and defects ${ }^{15}$ can be directly mapped across the investigated structure.

$\mathrm{BaTiO}_{3}$, one of the most typical perovskite ferroelectric materials, is widely used in multilayer ceramic capacitors ${ }^{\mathbf{1 6 , 1 7}}$ and many other applications. ${ }^{\mathbf{1 8 , 1 9}}$ The spontaneous polarization in $\mathrm{BaTiO}_{3}$ is originated from Ti off center displacement. ${ }^{20} \mathrm{Ca}$ ions has been an important chemical substitutions on Ba site for particular applications, such as lead free piezoelectric ${ }^{\mathbf{2 1 , 2 2}}$ or multilayer capacitors. ${ }^{23} \mathrm{Ca}$ could intriguingly remain the Curie temperature and enhance its polarization. ${ }^{24,25}$ It rebels the common principle that smaller A-site ions substituted into $\mathrm{BaTiO}_{3}$ matrix brings shrinkage of unit cell volume and suppresses the Ti displacement. ${ }^{26}$ Laterly, Ca off centering was putted forward, proved to have some similarities to $\mathrm{Pb}$ off centering, ${ }^{27}$ contributing to the polarization. ${ }^{28}$ The structurerefinement gave instantaneous atomic positions supported the ferroelectric enhancement in $(\mathrm{Ba}, \mathrm{Ca}) \mathrm{TiO}_{3} .{ }^{29}$ However, the precise atomic position determination of $\mathrm{Ti}$ displacement in $(\mathrm{Ba}, \mathrm{Ca}) \mathrm{TiO}_{3}$ materials at atomic scale is still lack.

Meanwhile, there is a remained controversy in $(\mathrm{Ba}, \mathrm{Ca}) \mathrm{TiO}_{3}$ system for decades. Many researchers have stated that $\mathrm{Ca}$ ions could occupy B-site, especially in non-stoichiometric design, or prepared with special method. ${ }^{30,31}$ The site occupancy of Ca ions is believed to significantly influence the enhanced dielectric properties, ${ }^{32,33}$ and Curie temperature, ${ }^{34}$ or even to induce double $P-E$ loops. ${ }^{35}$ To understand the local Ca site occupancy is of great importance for the drastically improvement of physical properties in $\mathrm{Ca}$ substituted $\mathrm{BaTiO}_{3}$ materials.

In the present work, we located atom positions from unit-cell scale in $\mathrm{BaTiO}_{3}$ and (Ba,Ca) $\mathrm{TiO}_{3}$ using high angle annular dark field (HADDF)-scanning transmission electron microscopy (STEM), equipped with electron energy loss spectroscopy
${ }^{a}$ Laboratory of Dielectric Materials, School of Materials Science and Engineering, Zhejiang University, Hangzhou 310027, China. E-mail: xmchen59@zju.edu.cn ${ }^{b}$ School of Materials Science and Engineering, Zhejiang University, Hangzhou 310027, China.E-mail: hetian@zju.edu.cn

$\dagger$ Electronic supplementary information (ESI) available. See DOI: $10.1039 / \mathrm{c} 7 \mathrm{ra00662d}$ 
(EELS), and annular bright field (ABF)-STEM. The displacement of $\mathrm{Ti}$ atom and oxygen octahedral with respect to A-site are determined and averaged in one single domain. Both Ti ions and oxygen octahedrons shift stronger with increasing $\mathrm{Ca}$ content. Besides, EELS signal of $\mathrm{Ca}$ ions at B-site is the first direct structure evidence of the $\mathrm{Ca}$ ions' occupancy of $\mathrm{Ti}$ ions.

The $(\mathrm{Ba}, \mathrm{Ca}) \mathrm{TiO}_{3}$ ceramics are prepared by a standard solid state reaction method. The reagent powders of $\mathrm{CaCO}_{3}(99.99 \%)$, $\mathrm{BaCO}_{3}(99.93 \%)$, and $\mathrm{TiO}_{2}(99.99 \%)$ were mixed, and the mixtures were calcined at $1273 \mathrm{~K}$ in air for $3 \mathrm{~h}$ after drying. The calcined powders were pressed into the disks of $12 \mathrm{~mm}$ in diameter and $2 \mathrm{~mm}$ in thickness under a uniaxial pressure of $98 \mathrm{MPa}$. The green compacts were sintered at $1623 \mathrm{~K}$ in air for $3 \mathrm{~h}$ to yield the dense ceramics. TEM samples were prepared from the grinded powder from ceramics. The XRD patterns are shown in Fig. S1. $\uparrow$ The selected two compositions in the present work: $\left(\mathrm{Ba}_{0.8} \mathrm{Ca}_{0.2}\right) \mathrm{TiO}_{3},\left(\mathrm{Ba}_{0.75} \mathrm{Ca}_{0.25}\right) \mathrm{TiO}_{3}$ are confirmed to be tetragonal phase (PDF card 05-0626). With increasing $\mathrm{Ca}$ content, the unit cell volume decreases due to smaller ion radius of $\mathrm{Ca}^{2+}$ than $\mathrm{Ba}^{2+}$, lead to the peak shift to higher angle. The preparation method, dielectric, ferroelectric properties has been comprehensively improved by $\mathrm{Ca}^{2+}$ substitution. ${ }^{24,25}$ The remanent polarization $P_{\mathrm{r}}$ and dielectric strength $E_{\mathrm{b}}$ are significantly enhanced by Ca-substitution, and the best $P_{\mathrm{r}}(11.34 \mu \mathrm{C}$ $\left.\mathrm{cm}^{-2}\right)$ and dielectric strength $E_{\mathrm{b}}\left(75 \mathrm{kV} \mathrm{cm}^{-1}\right)$ are obtained in the composition $x=0.25$. Thus, here in the present work we choose these best two compositions to illustrate this enhancement in microstructure structure. Plan view specimens for HRTEM are prepared by disaggregating the ceramic followed by grinding in an agate mortar. The powder was then suspended in acetone and dispersed onto a holey carbon 200 mesh TEM grid. The high-resolution HAADF-STEM image was acquired using a $G^{2}$ 80-200 Chemi STEM FEI Titan microscope fitted with an aberration corrector for the probe-forming lens, operated at 200 $\mathrm{kV}$, equipped with electron energy-loss spectroscopy (EELS). The STEM convergence semi-angle was approximately 21.4 mrad, providing a probe size of approximately $0.8 \AA$ at $200 \mathrm{kV}$.

Fig. 1(a) shows the HADDF-STEM image of $\left(\mathrm{Ba}_{0.75} \mathrm{Ca}_{0.25}\right) \mathrm{TiO}_{3}$ ceramic in a projection along [010] axis, similar HADDF-STEM image of $\left(\mathrm{Ba}_{0.80} \mathrm{Ca}_{0.20}\right) \mathrm{TiO}_{3}$ ceramic is shown in Fig. S2. $\dagger \mathrm{We}$ selected an area (the green rectangle) to do the EELS spectrum to observe the distribution of cations. By integrating the

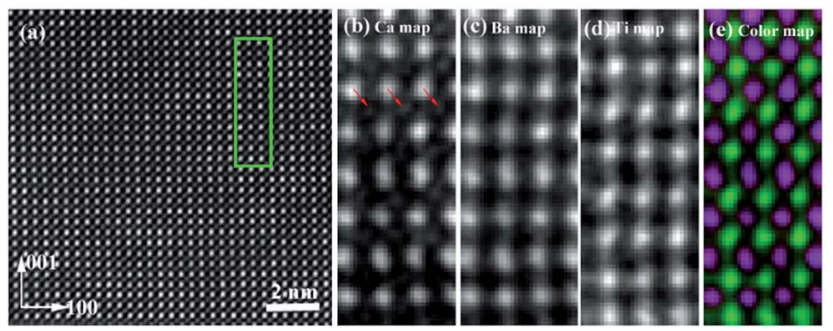

Fig. 1 (a) HAADF STEM image of $\mathrm{Ba}_{075} \mathrm{Ca}_{0.25} \mathrm{TiO}_{3}$ ceramics including the survey region $3 D$ EELS data is acquired from the region indicated by the green rectangle. The atomic resolution elemental maps of $\mathrm{Ca}$ (b), $\mathrm{Ba}(\mathrm{c})$ and $\mathrm{Ti}$ (d) are separately present. (e) Color map of the $\mathrm{Ca}$ (red), $\mathrm{Ti}$ (green) and $\mathrm{Ba}$ (blue) from the survey region. intensity of ionization edges from the atomic-resolution EELS spectra where the peak intensity is related to the elemental content, the distribution of specific cation can be mapped (see Fig. 1(b-e)). Ba ions signal in EELS spectra only appears at Asite, and Ti ions signal only appears at B-site. However, the doped $\mathrm{Ca}$ atoms show EELS signal at both $\mathrm{A}^{-}$and B-sites (indicated as the arrowheads in Fig. 1(b)). With atomic EELS spectrum image, each single pixel can be corresponded to an EELS spectrum. Several pixels corresponding to A-site atoms in the spectrum image were selected and fitted to a linear combination of $\mathrm{Ca}^{2+}$ and $\mathrm{Ba}^{2+}$ reference spectra using EELS MODEL, ${ }^{35}$ therefore, the average $\mathrm{Ca} / \mathrm{Ba}$ ratio of $\mathrm{A}$ site was obtained. The detailed image simulations of EELS spectrum demonstrate that about $15 \pm 2 \%$ of Ca atoms substitute A-site, the left $4 \pm 1 \% \mathrm{Ca}$ atoms are located at B-site in $\left(\mathrm{Ba}_{0.80} \mathrm{Ca}_{0.20}\right)$ $\mathrm{TiO}_{3}$. Similarly, only $18 \pm 2 \% \mathrm{Ca}$ atoms substitute A-site, and 7 $\pm 1 \%$ occupy B-site in $\left(\mathrm{Ba}_{0.75} \mathrm{Ca}_{0.25}\right) \mathrm{TiO}_{3}$. This $7 \pm 1 \%$ is a big portion that would influence the local strain field and build-in electric field, for the $\mathrm{Ca}$ ions radius and charge imbalance. It is confirmed here that the $\mathrm{Ca}$ ions could occupy B site with microstructure evidence for the first time. It is worth to notice that the large portion of Ca cations occupied B-site could influence lots of physical properties, such as lowering Curie temperature, and inducing double hysteresis loops. This is also the first demonstration of Ca occupancy at B-site in the composition near solid solution limit in $(\mathrm{Ba}, \mathrm{Ca}) \mathrm{TiO}_{3}$, which means the multisite occupancy of $\mathrm{Ca}$ happens across the whole solid solution within the solid solution limit.

To make sure the reliability of the occupancy of specific atoms, the image intensity must be free of contributions arising from electron scattering at neighboring atomic columns. In order to guarantee that the Ca EELS intensity at B-site is not from the scattering of the A-site Ca ions, we used STEM sim software to simulate the HADDF STEM image and EELS STEM mapping image of $\mathrm{Ba}_{0.75} \mathrm{Ca}_{0.25} \mathrm{TiO}_{3}$ along [010] zone axis which is presumably regarded only contains A-site Ca. The main parameters in Bloch wave modification are listed in Table S1. $\dagger$ The sample is regarded to keep at room temperature (295 K), with a thickness $20 \mathrm{~nm}$, and occupy a tetragonal $\mathrm{BaTiO}_{3}$ like phase. The crystal structure of the modification is shown in Fig. S3. $\dagger$ As shown in Fig. 2(a), the simulated HADDF-STEM image corroborates well to the experimental result in Fig. 1(a). Fig. 2(c-e) show the simulated STEM EELS image of $\mathrm{Ba}, \mathrm{Ca}$, and $\mathrm{Ti}$ atoms. It is worth noting that the simulated EELS image of $\mathrm{Ca}$ ions shows no signal at B-site (see Fig. 2(d)). As shown in Fig. 2(b), it compares the intensity profile of line scanning in Fig. 2(d) and the line intensity from experimental observed Ca EELS-STEM in Fig. 1(b). It is clearly that the simulated line intensity of Ca signal only shows a peak at A-site positions. However, the experimentally observed Ca EELS signal show intensity peak at B-site. This simulation excludes the possibility of $\mathrm{Ca}$ ions EELS signal at B-site due to the delocalization inelastic scattering of A-site $\mathrm{Ca}$ ions. That is to say, the experimental EELS signal of $\mathrm{Ca}$ ions at B-site shown in Fig. 1(b) is exactly due to the $\mathrm{Ca}$ ions occupied B-site, not the inelastic scattering from A-site $\mathrm{Ca}$ ions. Same simulation has also been performed in $\mathrm{Ba}_{0.80} \mathrm{Ca}_{0.20} \mathrm{TiO}_{3}$, which can be found in Fig. S4. $\dagger$ 


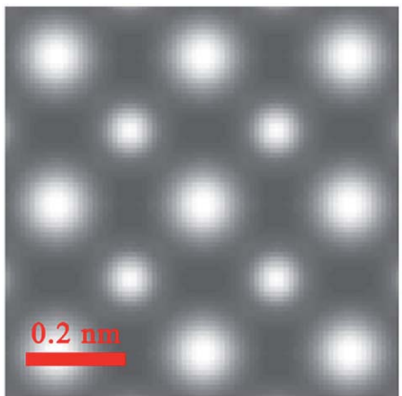

(a)

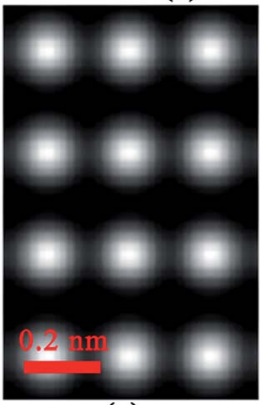

(c)

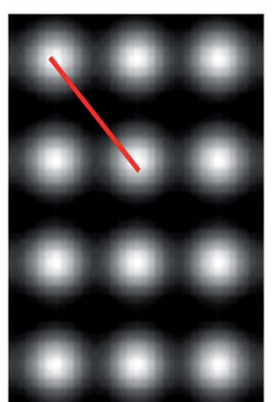

(d)

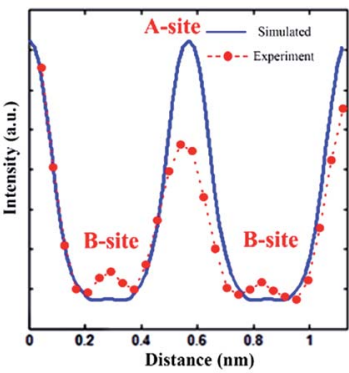

(b)

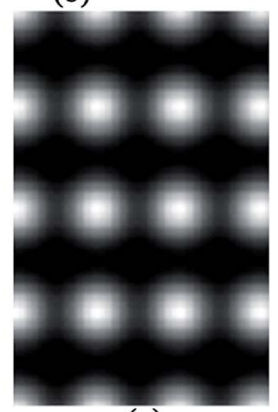

(e)
Fig. 2 Simulated results for $\mathrm{Ba}_{0.75} \mathrm{Ca}_{0.25} \mathrm{TiO}_{3}$ sample of (a) atomic resolution HAADF STEM image viewed along [100] zone axis (b) intensity profile of simulated (blue line) and experimental (red dots) EELS spectrum image of $\mathrm{Ca}$ along the indicated line in (d). Simulated STEM EELS image of (c) Ba (d) Ca (e) Ti with Gaussian peak filter of $0.07 \mathrm{~nm}$

The displacements of dipole ions in displacement ferroelectrics is of great importance to understand their polarization. For $(\mathrm{Ba}, \mathrm{Ca}) \mathrm{TiO}_{3}$, the enhancement in polarization compared to $\mathrm{BaTiO}_{3}$ has not been clarified from atomic displacements of its Ti-O dipoles, which should be most direct method to understand the enhancement. Here, the HADDF and ABF-STEM images have been combined to investigate the displacements of Ti-O dipoles (green HAADF image; red inverted ABF image), which are simultaneously obtained along [010] zone axis in $\mathrm{Ba}_{0.75} \mathrm{Ca}_{0.25} \mathrm{TiO}_{3}$. As shown in Fig. 3, the structure (one projected unit cell) is indicated schematically (position of $\mathrm{Ti}, \mathrm{Ba} / \mathrm{Ca}$, and $\mathrm{O}$ atomic columns are indicated by blue, green/yellow, and red circles, respectively). The position of $\mathrm{Ti}$ and $\mathrm{O}$ atoms displace along $c$ axis by a distance $d_{1}$ and $d_{2}$ with respect to the position of the center of Ba sites. This shift means the unit cell is in a polarized state.

For a long time, the precise observation of oxygen has been difficult. In TEM analysis, therefore, more attention has been paid to the contribution of displacement of metal ions for ferroelectricity, such as Ti ions in $\mathrm{BaTiO}_{3}$ and $\mathrm{PbTiO}_{3}$. Most of the local polarization has been calculated by the displacement of metal ions only. Oxygen octahedral displacement has been ignored or implied by empirical relation respect to $\mathrm{Ti}$ ions. However, oxygen octahedral usually has larger displacement than $\mathrm{Ti}$ ions in perovskites, and its direction dominantly determines the local polarization. In order to precisely determine the displacement of each atom, especially oxygen, annular bright field (ABF)-STEM was used to locate Ti-O dipoles. As

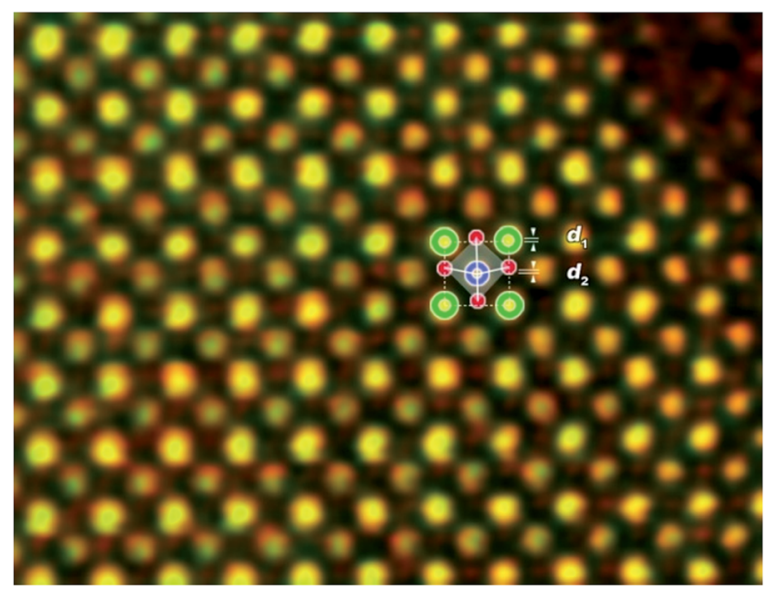

Fig. 3 Combined image of HAADF and simultaneously obtained ABFSTEM of the $\mathrm{Ba}_{0.75} \mathrm{Ca}_{0.25} \mathrm{TiO}_{3}$ view along [010] zone axis. The schematic view of the crystals structure of the tetragonal $\mathrm{Ba}_{0.75} \mathrm{Ca}_{0.25} \mathrm{TiO}_{3}$ is also shown. The $d_{1}$ and $d_{2}$ denote the displacement of Ti atoms (blue) and $\mathrm{O}$ atoms (red) along $\mathrm{c}$ axis from the centrosymmetry.

shown in Fig. 4, ABF-STEM images have been recorded along [010] zone axis. The darkest spots correspond to the mixture of heaviest $\mathrm{Ba}$ and $\mathrm{Ca}$ atoms, and the slightest spots refer to oxygen atoms. The atomic displacement map of $\mathrm{Ti}$ ions (Fig. 4(a)) and the center of oxygen octahedral (Fig. 4(b)) with respect to $\mathrm{Ba}$ ions center are modified, superimposed on the ABF-STEM image. The direction of the arrows indicates the polarization direction with respect to the center of $\mathrm{Ba}$ ions, and the color scale of arrows indicates the magnitude of polarization vector. The position of the contrast maxima in the images, (a)

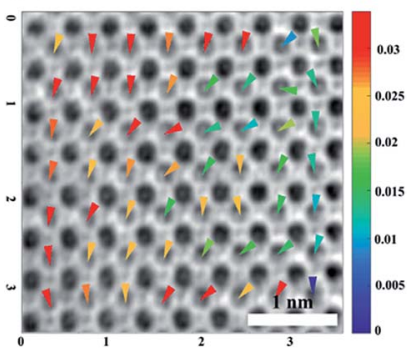

(c)

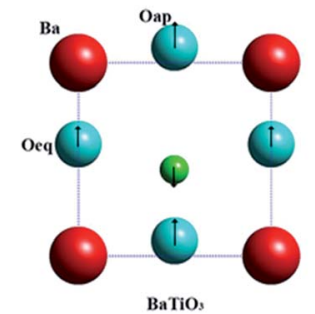

(b)

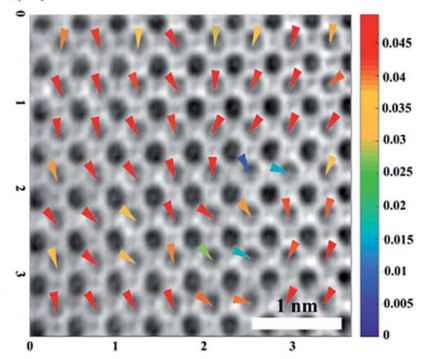

(d)

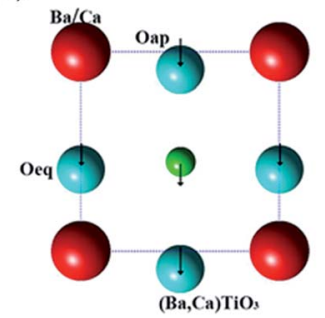

Fig. 4 Map of atomic displacement vectors showing: the displacement of (a) Ti atoms (arrows), and (b) the center of oxygen octahedral (arrows) from the center of the projected $\mathrm{Ba}$ atoms superposed on the ABF-STEM images [010] zone axis of $\mathrm{Ba}_{0.75} \mathrm{Ca}_{0.25} \mathrm{TiO}_{3}$. The plane view of $\mathrm{BaTiO}_{3}$ (c) and $(\mathrm{Ba}, \mathrm{Ca}) \mathrm{TiO}_{3}$ (d) projected from a axis. 
corresponding to the atomic positions, were determined by means of a least-squares fit of the intensity distribution using two-dimensional Gaussian profiles.

The Ti displacement map (Fig. 4(a)) superimposed on the ABF-STEM image illustrate a clear alignment of Ti upward along $c$ axis, coincided with the direction of spontaneous polarization in tetragonal $\mathrm{BaTiO}_{3}$. The oxygen octahedrons shift stronger in same direction compared to Ti ions. The average magnitude of the local $\mathrm{Ti}$ displacement in the present mono-domain is calculated as $0.263 \pm 0.111 \AA$, whereas the oxygen octahedron has an average displacement of $0.268 \pm 0.100 \AA$. The average displacement of $\mathrm{Ti}$ ions is much higher than that in the nano sized $\mathrm{BaTiO}_{3}$ value $(0.14 \AA) .{ }^{36}$

With further investigating the oxygen ions, we distinguish the three oxygen ions in one unit cell as one apical oxygen $\left(\mathrm{O}_{\mathrm{ap}}\right)$ and two equatorial oxygen $\left(\mathrm{O}_{\mathrm{eq}}\right)$. The average displacement of apical oxygen $\left(\mathrm{O}_{\mathrm{ap}}\right)$ is $0.264 \pm 0.110 \AA$, and the average displacement of equatorial oxygen $\left(\mathrm{O}_{\text {eq }}\right)$ is $0.347 \pm 0.145 \AA$. This value is much higher than that in $\mathrm{BaTiO}_{3}$ structure. According to the previous report of neutron diffraction in $\mathrm{BaTiO}_{3}$ structure, the Ti ions move upward around $0.0904 \AA$, whereas the equatorial oxygen and apical oxygen moves downward $0.0424 \AA$ and $0.0985 \AA$, respectively. ${ }^{37}$ As shown in Fig. 4(c and d), it can be compared that the displacements of $\mathrm{Ti}$ and $\mathrm{O}$ ions shifts to the same direction in $(\mathrm{Ba}, \mathrm{Ca}) \mathrm{TiO}_{3}$ whereas they shift to opposite direction in $\mathrm{BaTiO}_{3}$. It is similar to that in $\mathrm{PbTiO}_{3}$, wherein the $\mathrm{Ti}$ and $\mathrm{O}$ ions move to the same direction, and the displacements are much higher than that in $\mathrm{BaTiO}_{3}$. In this respect, the Ti-O dipoles in (Ba,Ca) TiO 3 has similarity to that in $\mathrm{PbTiO}_{3}$, and it should be the structure origin of the ferroelectric enhancement in $(\mathrm{Ba}, \mathrm{Ca}) \mathrm{TiO}_{3}$ compared to $\mathrm{BaTiO}_{3}$.

With these result, we can analysis the site contributions to the net polarizations using the equation below: ${ }^{38}$

$$
\delta P=\frac{e}{v} \sum_{m=1}^{N} Z_{m}^{*} \times \delta u_{m}
$$

where, $N$ is the number of atoms in the primitive unit cell, $\delta u_{m}$ is position vector, and $v$ is the volume of the unit cell. In the calculation, the center of $\mathrm{Ba} / \mathrm{Ca}$ is fixed as zero point and the effective charges for oxygen atoms are distinguished as two equatorial atoms and one apical atom. Disregarding the distortion of oxygen octahedrons, and the difference of Born effective charge in $\mathrm{Ba}$ or $\mathrm{Ca}$ ions, the spontaneous polarization of one unit cell in (Ba,Ca) $\mathrm{TiO}_{3}$ can be calculated as follow:

$$
P_{\mathrm{s}}=\frac{e}{v}\left(2 \delta_{\mathrm{eq}} Z_{\mathrm{eq}}+\delta_{\mathrm{ap}} Z_{\mathrm{ap}}-\delta_{\mathrm{Ti}} Z_{\mathrm{Ti}}\right)
$$

where, $Z_{\text {eq }}, Z_{\text {ap }}$, and $Z_{\mathrm{Ti}}$ are the Born effective charge of equatorial oxygen and apical oxygen and $\mathrm{Ti}$ atom, respectively. The $\delta_{\text {eq }}, \delta_{\text {ap }}$, and $\delta_{\mathrm{Ti}}$ are the displacement of atom from the positions of centrosymmetry, respectively. The $v$ denotes the unit cell volume, which is refined from XRD result. The data are obtained from average displacements from multiple single domains. The local average polarization of the single domain is calculated as $27.6 \mu \mathrm{C} \mathrm{cm}^{-2}$, which is slightly higher than the macroscopic polarization measured in ceramic sample. It is due to the defects and different polarization direction in multiple $90^{\circ}$ and $180^{\circ}$ domains in ceramic sample.

\section{Conclusions}

In summary, in $\left(\mathrm{Ba}_{0.75} \mathrm{Ca}_{0.25}\right) \mathrm{TiO}_{3}$ ceramic, a big portion of $\sim 7 \%$ Ca content at B-site is confirmed by EELS-STEM, which is the first TEM evidence for Ca ions' B-site occupation. This founding is very important to understand the Curie temperature, dielectric constant and double ferroelectric hysteresis loops in (Ba,Ca) $\mathrm{TiO}_{3}$ ceramics. More importantly, average Ti displacement of $0.263 \AA$, and large apical and equatorial oxygen displacement of $0.264 \AA$ and $0.347 \AA$ were confirmed using HADDF-STEM combined with ABF-STEM in $\left(\mathrm{Ba}_{0.75} \mathrm{Ca}_{0.25}\right) \mathrm{TiO}_{3}$ single domain, respectively. This great increase of magnitude of displacements in $(\mathrm{Ba}, \mathrm{Ca}) \mathrm{TiO}_{3}$ compared to $\mathrm{BaTiO}_{3}$ is the inherent structure origin of the ferroelectric enhancement in $\mathrm{Ca}$ substituted $\mathrm{BaTiO}_{3}$.

\section{Acknowledgements}

The present work was supported by National Natural Science Foundation of China under grant numbers 51332006 and Chinese National Basic Research Program under grant number 2015 CB654601.

\section{Notes and references}

1 J. F. Scott, Science, 2007, 315, 954.

2 S. A. Bhalla, R. Guo and R. Roy, Mater. Res. Innovations, 2016, 4, 3 .

3 S. A. Gridnev, Ferroelectrics, 2002, 266, 171.

4 S. C. Abrahams, S. K. Kurtz and P. B. Jamieson, Phys. Rev., 1968, 172, 551.

5 C. H. Ahn, K. M. Rabe and J. M. Triscone, Science, 2004, 303, 488.

6 J. Seidel, L. W. Martin, Q. He, Q. Zhan, Y. H. Chu, A. Rother, M. E. Hawkridge, P. Maksymovych, P. Yu, M. Gajek, N. Balke, S. V. Kalinin, S. Gemming, F. Wang, G. Catalan, J. F. Scott, N. A. Spaldin, J. Orenstein and R. Ramesh, Nat. Mater., 2009, 8, 229.

7 C. L. Jia and K. Urban, Science, 2004, 303, 2001.

8 C. Ma, Y. Lin, H. Yang, H. Tian, L. Shi, J. Zeng and J. Li, Adv. Mater., 2015, 27, 6328.

9 S. D. Findlay, N. Shibata, H. Sawada, E. Okunishi, Y. Kondo, T. Yamamoto and Y. Ikuhara, Appl. Phys. Lett., 2009, 95, 191913.

10 C.-L. Jia, V. Nagarajan, J.-Q. He, L. Houben, T. Zhao, R. Ramesh, K. Urban and R. Waser, Nat. Mater., 2007, 6, 64. 11 C.-L. Jia, S.-B. Mi, K. Urban, I. Vrejoiu, M. Alexe and D. Hesse, Nat. Mater., 2008, 7, 57.

12 C.-L. Jia, K. W. Urban, M. Alexe, D. Hesse and I. Vrejoiu, Science, 2011, 331, 1420.

13 C. T. Nelson, B. Winchester, Y. Zhang, S. J. Kim, A. Melville, C. Adamo, C. M. Folkman, S. H. Baek, C. B. Eom, D. G. Schlom, L. Q. Chen and X. Pan, Nano Lett., 2011, 11, 828. 
14 S.-B. Mi, C.-L. Jia, I. Vrejoiu, M. Alexe and D. Hesse, Adv. Mater. Interfaces, 2015, $2,8$.

15 F. Azough, D. Kepaptsoglou, Q. M. Ramasse, B. Schaffer and R. Freer, Chem. Mater., 2015, 27, 497.

16 K. Hiroshi, M. Youichi and C. Hirokazu, Jpn. J. Appl. Phys., 2003, 42, 1.

17 Y. Sakabe, Curr. Opin. Solid State Mater. Sci., 1997, 2, 584.

18 E. Burcsu, G. Ravichandran and K. Bhattacharya, Appl. Phys. Lett., 2000, 77, 1698.

19 T. Hirofumi, N. Yoshiki, T. Junji, M. Kazuya, Q. Jinhao and T. Sadahiro, Jpn. J. Appl. Phys., 2006, 45, L30.

20 B. Ravel, E. A. Stern, R. I. Vedrinskii and V. Kraizman, Ferroelectrics, 1998, 206, 407.

21 W. Liu and X. Ren, Phys. Rev. Lett., 2009, 103, 257602.

22 B. Li, J. E. Blendell, K. J. Bowman and M. Hoffmann, J. Am. Ceram. Soc., 2011, 94, 3192.

23 G. Yao, X. Wang, T. Sun and L. Li, J. Am. Ceram. Soc., 2011, 94, 3856.

24 X. N. Zhu, W. Zhang and X. M. Chen, AIP Adv., 2013, 3, 082125.

25 D. Fu, M. Itoh, S.-y. Koshihara, T. Kosugi and S. Tsuneyuki, Phys. Rev. Lett., 2008, 100, 227601.

26 V. V. Lemanov, E. P. Smirnova, P. P. Syrnikov and E. A. Tarakanov, Phys. Rev. B: Condens. Matter Mater. Phys., 1996, 54, 3151.

27 R. E. Cohen, Nature, 1992, 358, 136.
28 V. Krayzman, I. Levin, J. C. Woicik, F. Bridges, E. J. Nelson and D. C. Sinclair, J. Appl. Phys., 2013, 113, 044106.

29 I. Levin, V. Krayzman and J. C. Woicik, Appl. Phys. Lett., 2013, 102, 162906.

30 P. S. R. Krishna, D. Pandey, V. S. Tiwari, R. Chakravarthy and B. A. Dasannacharya, Appl. Phys. Lett., 1993, 62, 231.

31 S. Lee and C. A. Randall, Appl. Phys. Lett., 2008, 92, 111904. 32 S.-H. Yoon, S.-H. Kang, S.-H. Kwon and K.-H. Hur, J. Mater. Res., 2011, 25, 2135.

33 Y. Sakabe, N. Wada, T. Hiramatsu and T. Tonogaki, Jpn. J. Appl. Phys., 2002, 41, 6922.

34 V. S. Puli, D. K. Pradhan, B. C. Riggs, D. B. Chrisey and R. S. Katiyar, J. Alloys Compd., 2014, 584, 369.

35 F. Peña, R. Arenal, O. Stephan, M. Walls, A. Loiseau and C. Colliex, in EMC 2008 14th European Microscopy Congress, 1-5 September 2008, Aachen, Germany: Volume 1: Instrumentation and Methods, ed. M. Luysberg, K. Tillmann, and T. Weirich, Springer Berlin Heidelberg, Berlin, Heidelberg, 2008, p. 367.

36 M. B. Smith, K. Page, T. Siegrist, P. L. Redmond, E. C. Walter, R. Seshadri, L. E. Brus and M. L. Steigerwald, J. Am. Chem. Soc., 2008, 130, 6955.

37 G. H. Kwei, A. C. Lawson, S. J. L. Billinge and S. W. Cheong, J. Phys. Chem., 1993, 97, 2368.

38 W. Zhong, R. D. King-Smith and D. Vanderbilt, Phys. Rev. Lett., 1994, 72, 3618. 